\title{
The influence of traffic flow dynamics on urban soundscapes
}

\author{
Bert De Coensel ${ }^{\mathrm{a}, *}$, Tom De Muer ${ }^{\mathrm{a}}$, \\ Isaak Yperman ${ }^{b}$, Dick Botteldooren ${ }^{a}$ \\ ${ }^{a}$ Acoustics Group, Department of Information Technology, Ghent University, St. Pietersnieuwstraat 41, \\ B-9000 Ghent, Belgium \\ b Transportation Planning and Highway Engineering Group, Department of Civil Engineering, \\ Katholieke Universiteit Leuven, Kasteelpark Arenberg 40, B-3001 Heverlee, Belgium
}

Available online 15 September 2004

\begin{abstract}
The development and validation of a model for dynamic traffic noise prediction is presented. The model is composed of a GIS-based traffic microsimulation part coupled with an emission model, and a beamtrace-based $2.5 \mathrm{D}$ propagation part, which takes into account multiple reflections and diffractions. The model can be used to analyze the influence of real urban traffic situations (e.g., traffic flow management, road saturation) in the usual equivalent sound level maps. However, it also allows to calculate and visualize statistical noise levels and indicators derived from them. Novel descriptors based on the power spectrum of noise level fluctuations can be obtained. A part of Gentbrugge, Belgium, is taken as a validation area; different traffic demand scenarios are simulated.
\end{abstract}

(c) 2004 Elsevier Ltd. All rights reserved.

Keywords: Traffic flow dynamics; Microsimulation; Acoustic beamtrace; Urban soundscape

\footnotetext{
* Corresponding author. Tel.: +32 92649995; fax: +3292649960.

E-mail address: bert.decoensel@intec.ugent.be (B. De Coensel).
} 


\section{Introduction}

To guarantee mobility while minimizing its negative impact on man and environment, is one of the main challenges for sustainable development of our society. Although the interest in the study of traffic dynamics is more than 50 years old [1] and in spite of many efforts to cope with the ever growing mobility demand, in the last decades the traffic situation has deteriorated a lot. The time that drivers spend standing in traffic jams amounts to several days each year; vehicle emissions have reached or even exceeded a level comparable to those by industrial production and those by private households.

An important consequence, particularly but not only in urban environment, is disturbance by noise. The assessment of the impact of mobility on the urban sound climate and the quality of life, is mostly based on the calculation of noise maps, using a set of standard calculation schemes for the evaluation of the contribution of different sources. This has already been extended to mapping the expected percentage of highly annoyed people based on exposure-response relationships [2]; there have even been initiatives to produce an integrated presentation of different environmental and quality of life-maps $[3,4]$.

However, today mainstream methods are mostly based on the measurement and prediction of time-averaged A-weighted levels and derived measures such as $L_{\mathrm{dn}}$ or $L_{\mathrm{den}}$, which account for the different response of people to noise during the evening $(+5 \mathrm{~dB}$ correction) and the night $(+10 \mathrm{~dB})$. Traffic is hereby modelled as a steady sound source flow. There is a consensus that road traffic noise causes annoyance, but some studies have detected unexplained peaks of annoyance in quieter places, or a plateau in the relation between annoyance and noise at high noise levels (an overview can be found in [5]). The time pattern of the noise of vehicles passing by may explain these anomalies. In [6], a principal components analysis revealed three factors describing at best variations in auditory judgment on various urban situations, one of which was strongly correlated with the time variations of the noise. One way to modify the dynamics of passby sound, is to concentrate on the sound quality of individual vehicles. The SVEN project [7-9], for example, is looking at a number of aspects of vehicle noise, developing methods for the objective and subjective assessment of traffic noise quality. However, recent studies have shown that there are also clear differences in longer-term dynamics between different urban settings, caused by the alternation of passby noise and background noise $[10,11]$.

An important tool to change traffic dynamics, and as a consequence to change traffic noise dynamics, is traffic flow management. In the last few years, there has been a considerable amount of effort spent on the study of the impact of traffic light timing [12,13], road bumps [14] and roundabouts [15,16] on overall noise immission levels. These studies were of theoretical as well as of experimental nature. The need for traffic noise prediction models which are able to represent interrupted and complex flow is stressed in $[17,18]$.

The introduction of the time component in traffic noise prediction poses an additional problem: what are suitable indicators? In an attempt to assess the annoyance caused by fluctuating noise, several descriptors have been proposed [19], such as 
$L_{5}-L_{95}, N_{5}-N_{95}$ or $L_{10}-L_{90}$. The traffic noise index TNI $=4\left(L_{10}-L_{90}\right)+L_{90}-30$ proposed in [20] and the noise pollution level $L_{\mathrm{NP}}=L_{\mathrm{eq}}+k \sigma$ (where $k$ is a constant and $\sigma$ is the standard deviation of the sound level) introduced in [21] are encountered occasionally. These indicators all give an idea about the size of the fluctuations from the average background noise.

However, almost exclusively only the negative aspects of the urban sound climate are nowadays taken into account (annoyance, stress, heart diseases, etc.), while the positive axis in the analysis of soundscapes is seldom considered. Sometimes, it is not desirable to simply make the environment quieter, because some urban areas ask for a matching sound, e.g., the sound of birds in a city park or the hum of a market place [22]. From the soundscape point of view, it looks appealing to evaluate the patterns in time variations also. A way to do this is to calculate the power spectrum of sound amplitude fluctuations. This was first done for music already in the late 1970s [23,24], and has recently been adopted to environmental soundscape research [25].

This paper will present a tool for dynamic traffic noise prediction. The starting point will be the GIS-based microsimulation of the traffic in an urban neighbourhood or part of a town. Compared to the earlier work [12-16] that concentrated on one intersection or one street, this has the advantage that a much more realistic traffic dynamics situation can be created, and that sources at a greater distance also can contribute. This microsimulation model will be coupled with a vehicle emission model and a state of the art propagation model based on acoustic beamtrace methods, which takes into account multiple reflections and diffractions. This way, the soundfield at the quiet sides of buildings or in urban quiet areas can be simulated accurately. The result will be a time series of immission values at different observer points. These will allow to draw maps of the $L_{\text {Aeq }}$ and statistical levels $L_{5}, L_{50}, L_{95}$, etc., but also to make maps of dynamic descriptors based on the power spectrum of noise amplitude fluctuations. The proposed model will be compared to measurements in Section 3 and it will be illustrated how the model can be used in Section 4.

\section{Methodology}

\subsection{Traffic modelling}

Since the 1950s, there is a clear evolution in the modelling of road traffic [26]. The early models were based on a fluid-dynamic model for kinematic waves [27], which made it possible to simulate the propagation of density jumps in traffic; later microscopic follow-the-leader models [28] and gas-kinetic, or so called Boltzmann-like models [29] were introduced. In the last decade, discrete cellular automata models of vehicle traffic [30,31], shortly called micromodels, have become very popular, mainly because of the large computing power that has become available.

In these micromodels each vehicle, represented by a particle, is simulated individually. The nature of the interactions between these particles is determined by the way vehicles influence each others movement. This way, traffic is simulated not as a steady flow, but as a system of interacting particles, far beyond equilibrium. The vehicle 
positions, speeds, velocities and accelerations as well as the simulated time are discretized.

The traffic noise prediction model that is developed, uses Paramics [32], a commercially available microsimulation package. The basic building blocks of Paramics road networks are nodes, corresponding to junctions, and links, which can be subdivided into several lanes. These can be coded starting from an overlay photograph. Different links and nodes can be grouped into zones, each representing a certain activity, like residential areas, industrial zones, parking lots, etc. During simulation, vehicles are created at random, are given an origin and destination zone pair, and are loaded onto the network on a link inside its origin zone. After reaching their destination zone they are cleared from the network. The demands between different zones are described by a so called origin-destination matrix (OD matrix). These OD matrices can be vehicle type specific to represent the actual vehicle fleet properties; they can also vary in time. Customization of the available vehicle types makes it possible to simulate cars, light and heavy goods vehicles, coaches, minibusses and busses. When seperate links and nodes are used, it is also possible to simulate the movement of trams and trains.

\subsection{Vehicle noise emission model}

Paramics allows users to write plugins, consisting of a dynamic link library bundling a set of callbacks, each called at defined points in the simulation. This makes it possible to extend and refine the microsimulation model. For our traffic noise prediction model a vehicle noise emission plugin was written. First of all, a viewport is set, which consists of a polygonal part of the network around the observer(s). Only vehicles inside this viewport will be taken into account. At each timestep, positional data of each vehicle inside the viewport is gathered, together with vehicle type, age, speed, acceleration, travelling direction and additional info about the link the vehicle is travelling on (e.g., the road surface type, the link gradient, etc.).

With each vehicle, one or more sources are associated, each consisting of an ISO octave band spectrum with center frequencies from $63 \mathrm{~Hz}$ to $8 \mathrm{kHz}$. Emission spectra are then calculated from an external database, and can be a function of the vehicle and link variables given in the previous paragraph. Currently the Nord 2000 vehicle noise emission database is used [33,34]. In this calculation scheme, a single directional source spectrum is associated with each vehicle. The source is located at the right-hand side of the vehicle at a height of $20 \mathrm{~cm}$. Emission values are only function of vehicle type and speed (in the form of tables), and only three emission classes are used: cars (CAR), dual-axle heavy vehicles (DHV) and multi-axle heavy vehicles (MHV). However, the design used makes it easy to extend the emission model, as data becomes available. Possible extensions could be the subdivision of vehicle emission into engine noise, tire/road noise and exhaust noise with associated sources, or the use of corrections for vehicle acceleration/deceleration, link gradient and road surface type.

Subsequently, the sources associated with each vehicle are mapped on a set of emission points. Two configurations are possible: 
1. A grid of emission points is placed on the part of the network inside the viewport. The links are segmented per lane with a user-defined segment length; one emission point is placed in the center of each segment. On the nodes a rectangular grid of emission points is placed. This allows to account for wide and complex crossings and for small roundabouts. During simulation, the vehicle sources are mapped to the nearest emission point. When the distance between adjacent emission points is large, it is possible that multiple sources are mapped to the same emission point, in which case the sum of the sound power spectra is calculated. It is also possible that vehicles travelling in different directions are mapped to the same emission point, so in this configuration the directional info cannot be taken into account.

2. A set of emission points is constructed on the fly for each vehicle in the network, at their exact locations. This way, directional info can be used. However, the positions of the emission points are changed each simulation timestep, which is a major disadvantage when it comes to propagation calculations, as discussed below.

\subsection{Propagation}

Once a set of time-changing vehicle noise emissions are available, dynamic noise immission at a set of observers can be calculated using a propagation model, specially tuned for time varying sources. The model implemented consists of 3 steps: path generation, attenuation calculation and immission calculation.

A beam tracing model is used to generate paths between the emission points and the receivers. The technique used is object precise polygonal beam tracing [35,36]. A beam consists of a group of rays, coherent in space (following about the same path) and bounded by the objects in the simulation area. The model uses a $2.5 \mathrm{D}$ representation of the world, which consists of a terrain model with super-positioned blocks representing the buildings (Fig. 1,2.5D). When looked from above, the world is seen as a number of polygons in a plane. Standing inside the world all walls are upright and all roofs are flat. Terrain and building data are loaded from standard GIS maps. The most important advantages of the beam tracing approach in comparison with ray tracing is that no receivers are missed by the infinitesimal small rays constituting the beams, and that diffraction is modeled efficiently, which is important in high shielded regions like urban areas.

The path generation itself is split into two parts. First, a set of beams is traced through the geometric network in 2D using the footprints of the houses as objects (Fig. 1,2D). The shaded areas in this figure represent the bundles. Second, paths are constructed between each emission point and receiver within reasonable distance, and the different diffraction and reflection points in a vertical section are computed (Fig. 1,0.5D).

While the beams propagate through the geometrical environment, they reflect and diffract on boundaries, so each beam must have a local view on the environment to efficiently perform this tracing. A convenient method to allow this is to use a convex cell subdivision of the environment $[37,38]$. Within each convex cell a beam has full 

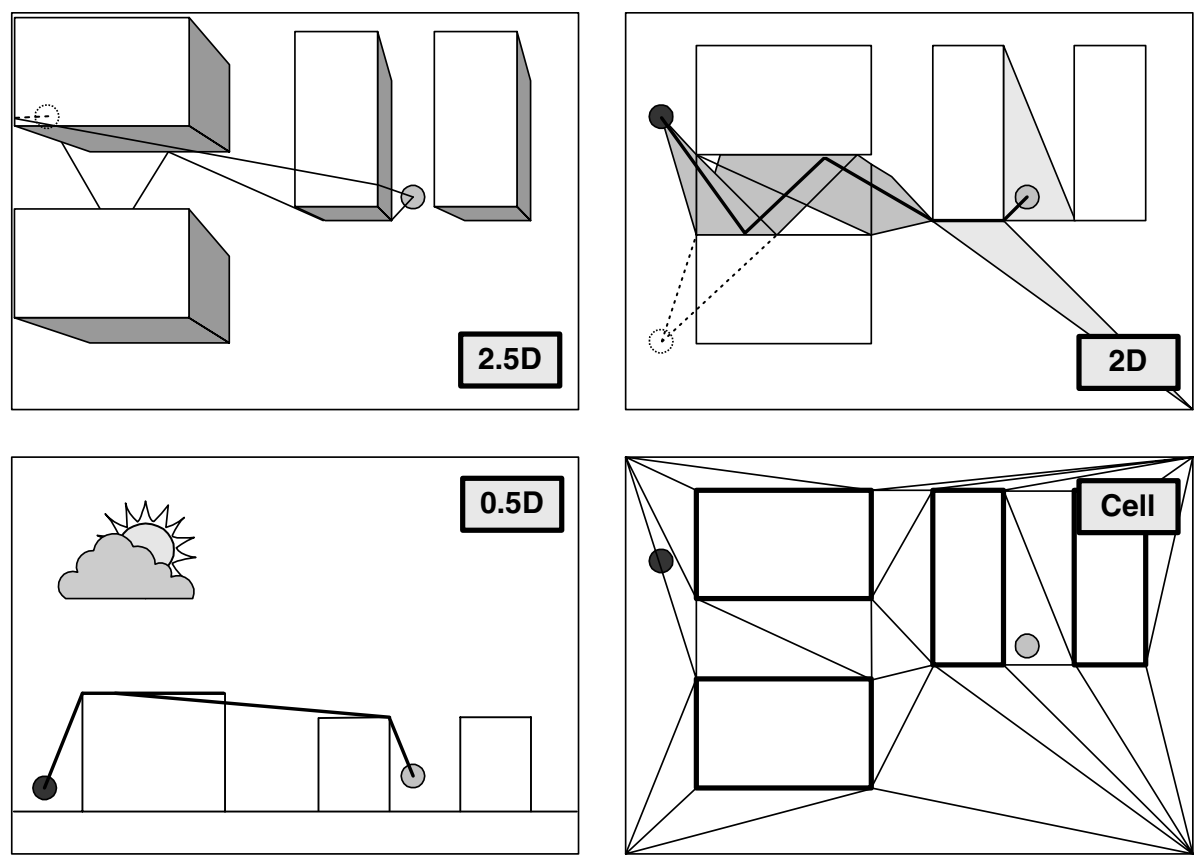

Fig. 1. Beam tracing through a $2.5 \mathrm{D}$ environment. The emission point is shown as a dark grey circle, the receiver as a light grey circle.

visibility by definition and construction. Thus, only interactions of a beam with a cell boundary have to be described. This cell boundary can be a wall of the real world environment or a portal, which is a virtual boundary placed during the convex cell construction. Fig. 1, cell shows a convex cell division where each cell has exactly three boundaries, also called a triangulation. The constrained Delaunay triangulation scheme is used, which preserves the original boundaries (walls of buildings) and has among all triangulation schemes the largest internal angle for all triangles, which is important for the numeric stability of the implementation. Using a triangulation over a convex polygonalization has the advantage that operations on the beams are easy to formalize and to implement. The disadvantage is that more beams need to be traced.

Once a beam is constructed and a receiver is detected inside it, the path between the emission point and the receiver is generated. The stack of beams is unwound and at each real boundary the points of interest (reflection and diffraction points) are computed on the fly. On modern pc's $(2 \mathrm{GHz})$ rates of 800,000 paths/s are achieved for the simplest configuration down to 200,000 paths/s when multiple reflections and diffractions are allowed, which is more than an order of magnitude faster than the actual attenuation computation following it. Diffuse reflections are not taken into account in the model. These can not be implemented optimally using a beamtracing technique; radiosity methods are more suited for this. A hybrid model would ask for considerably more computing time and resources. 
The attenuation model used is based on the ISO 9613 model [39], and has been extended with sideways diffraction according to the Nord 2000 model [40]. This model allows to take into account geometric divergence, atmospheric attenuation, ground effects and meteo effects (moderate downward refraction according to ISO 9613).

Finally, the immission at the receiver points is calculated by multiplying the emission with the attenuation coefficients. Two scenario's are possible, based on the emission point configuration (see Section 2.2). When exact source positions are used as emission points, the whole propagation calculation (path generation and attenuation calculation) has to be performed at each timestep. When a fixed grid of emission points is used, the propagation is done only once, and the attenuation between each emission point and receiver pair is stored. This way, it is possible to rapidly compute immissions for a series of timesteps. The downside is that more memory is needed for the calculations because there are more emission points, and thus more paths between emission points and receivers.

For the emission points in the vicinity of receivers the source direction is important, so the first scenario using exact vehicle positions has to be used. Of both scenarios, this provides the most accurate results. However, the duration of the attenuation calculation increases with the number of paths between the emission points and the receivers, which is about proportional to the square of the viewport radius, and also with the maximum number of reflections and diffractions allowed. For a typical urban setting of one receiver point and a viewport radius of $150 \mathrm{~m}$, enclosing about 10 sources, the propagation calculation has a duration of about $0.5 \mathrm{~s}$, taking into account four reflections and one diffraction. When 10,000 receiver points are considered, this can grow to $30 \mathrm{~s}$, resulting in a $15 \mathrm{~h}$ simulation time for a 15 min traffic simulation using a timestep of $0.5 \mathrm{~s}$.

When the simulation area is large, or there is a lot of traffic, it is no longer possible to simulate all vehicle sources using exact positions within a reasonable amount of time. In this case a combination of both scenarios has to be used, where vehicle sources at a larger distance from the observers are mapped to an emission point grid. Paths between receivers and sources at a greater distance are more complex, consisting of multiple reflections and diffractions, particularly in urban environment. A direct path is mostly not possible, so the source direction can be neglected for these sources. In Section 3.3 this approach is demonstrated. The grid used in this example has about 12,000 emission points; using the same propagation settings as in the previous paragraph, the propagation calculation has a duration of about $15 \mathrm{~min}$. Subsequent immission calculations using the attenuation coefficients stored take only about $50 \mathrm{~ms}$.

When a large number of observers is placed, e.g., for the calculation of a noise map, memory restrictions exist on the use of a grid, and it is more feasible only to use the first scenario, but making the viewport radius larger. This approach is illustrated in Sections 3.4 and 4.

Fig. 2 gives an overview of the complete dynamic noise immission model. 


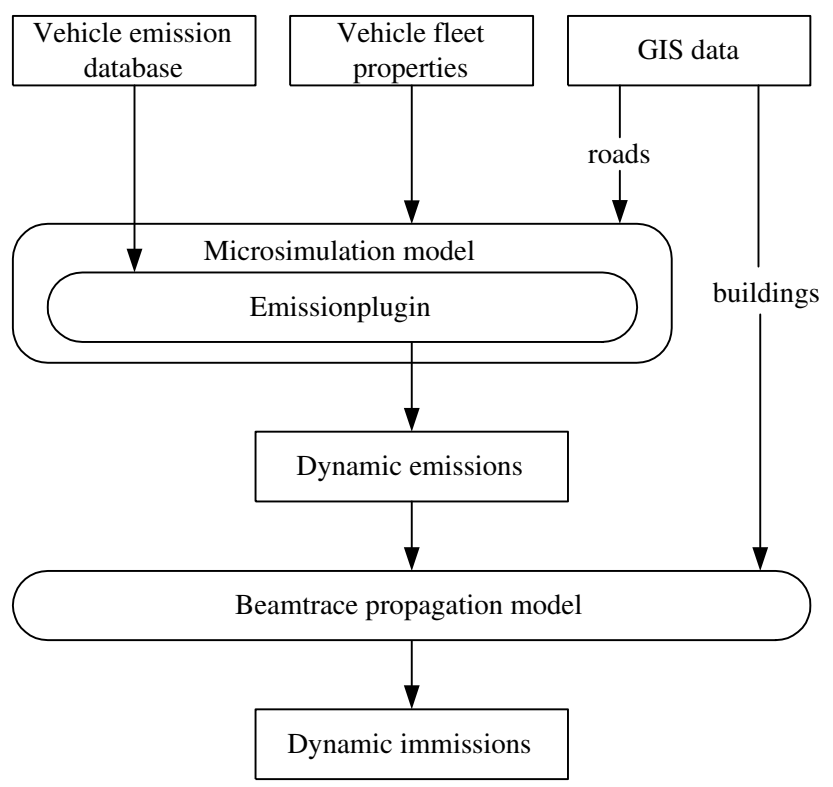

Fig. 2. Methodology outline.

\subsection{Impact analysis}

Dynamic noise immission calculations result in an enormous amount of raw data. To present these data in a condensed form in maps or tables, suitable indicators or descriptors have to be selected. Ideally these indicators reflect current knowledge on the impact on health, quality of life (annoyance) and general appreciation of the soundscape. Unfortunately, at least for health and annoyance effects, most recent research has focused on long term averaged noise levels $\left(L_{\mathrm{dn}}, L_{\mathrm{den}}\right)$ not explicitly taking into account fluctuation in noise exposure level.

For traffic noise $L_{\text {Aeq }}$ has become the measure most commonly used. In the past, other descriptors have also been suggested, based on the common assumption that fluctuating noise is more annoying, such as TNI and $L_{\mathrm{NP}}$, as mentioned in the introduction. The statistical level $L_{10}$ itself has also been used to characterize traffic noise annoyance [41]. Positive soundscapes, in particular quiet areas, are probably not well characterized by average noise levels. In [42] statistical noise levels, in particular $L_{95}$ and $L_{50}$, were proven to be much better discriminators between rural soundscapes subjectively evaluated as silent and those evaluated as not silent, than $L_{\text {Aeq }}$.

All the indices mentioned above have in common that they do not consider the time pattern of the exposure. A few long noise events separated by long periods of relative silence may result in the same statistical levels as a large number of short events separated by short periods of silence. In particular for the evaluation of soundscape quality, it can be important to distinguish between the above-illustrated situations by using a suitable indicator. It is proposed to use the spectrum of level 
fluctuations as an indicator. To calculate this spectrum, an $L_{\mathrm{Aeq}}, 1 \mathrm{~s}$ time-series of sufficiently long duration is necessary, e.g., $15 \mathrm{~min}$. The spectral density of this time-series is then calculated using an FFT. Because fluctuations in the sound level are considered, the corresponding frequencies are much lower, as opposed to the more usual spectrum of the instantaneous sound pressure. In this spectrum, periodic events will show up as peaks. When, e.g., a vehicle passes about each $10 \mathrm{~s}$, one will see a peak at about $0.1 \mathrm{~Hz}$ in this spectrum.

However, this spectrum has much more interesting characteristics. If the events contributing to the soundscape result from a complex system, then the spectrum will be linear on a log-log scale for the relevant inter-event time scales $(0.002-0.2 \mathrm{~Hz}$, or from $5 \mathrm{~s}$ to about $10 \mathrm{~min}$ ), according to the formula $f^{\alpha}$, with $f$ the frequency of level fluctuations. Self-organization of the underlying system will lead to a slope $\alpha=-1$, so called $1 / f$ behaviour. Steeper slopes $(\alpha<-1)$ tend to indicate high predictability; less steep slopes $(\alpha>-1)$ are an indication of a chaotic process. It was observed that $1 / f$ spectral characteristics are quite common in rural, natural, and urban soundscapes with a mixture of activities [25]. By drawing the link to music, where this temporal structure was observed earlier [23,24], it is suggested that perception of soundscape dynamics and the spectrum of sound level fluctuations are related and that the slope $\alpha$ of the spectrum and its deviation from linearity $\epsilon$ on a log-log scale, may be suitable soundscape descriptors [10]. To estimate $\alpha$, a linear fit of the spectrum is made in the interval from $0.002 \mathrm{~Hz}$ to $0.2 \mathrm{~Hz}$.

Based on the above analysis, it is proposed to use $L_{\text {Aeq }}$, statistical noise levels and in particular $L_{5}-L_{95}$, and the slope $\alpha$ of the spectrum of sound level fluctuations as primary descriptors that summarize the dynamics of the sound field.

\section{Case study: Gentbrugge}

\subsection{Traffic modelling and calibration}

To make a validation of the model presented, a part of Gentbrugge - a suburban area near Ghent, Belgium - was chosen as a study area. Fig. 3 shows the streets and the buildings in that area. The area contains local streets with low and medium amounts of traffic and a district road connecting the city of Ghent (located to the north-west) with other suburban areas. The E17 highway is crossing the area in the south, and is situated on a viaduct about $20 \mathrm{~m}$ high, with noise barriers on both sides. A railroad is also crossing the area, but is not shown in the figure (for the time being, the emission model only accounts for road traffic). The area, which has an area of about $1 \mathrm{~km}^{2}$, has a mainly residential use; almost no industry is located in it. Road traffic and daily life of the inhabitants are the main sources of noise. Coding of the area in Paramics resulted in a network consisting of about 180 nodes and 450 links. Maximum vehicle speeds on the urban roads varied from 45 to $70 \mathrm{~km} / \mathrm{h}$ (note that these are not the legal speed limits); for the highway it was $120 \mathrm{~km} / \mathrm{h}$. Turning restrictions and priorities for the junctions were set as in reality, as well as the signal timing for the three signalized junctions shown in Fig. 3. 


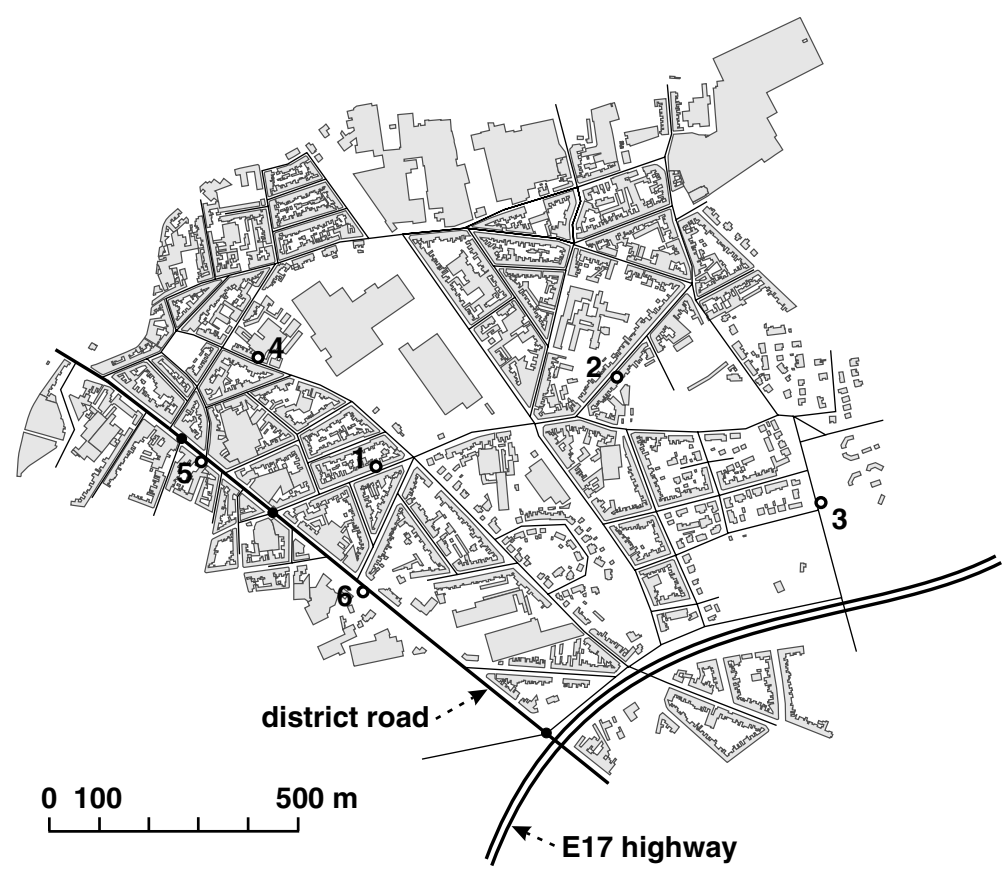

Fig. 3. View of the study area Gentbrugge. In grey, the buildings are shown; the black lines give an impression about the road network. The district road at the south-west is shown in bold, with the three signalized junctions shown as black dots. At the south-east, the E17 highway is shown; six observer points are shown with a circle at the measurement side of the road.

To be able to realistically model the traffic flows in the network, correct traffic data was necessary. Different simulations with the Macroscopic Traffic Model of the City of Ghent gave a rough image of the origins and destinations of vehicles on the major roads passing through our network, during the morning and evening rush-hour. For these periods, the number of vehicles passing per hour on the major roads were also calculated using this model. Subsequently, on three locations within the study area, traffic counts were done by the Roads and Traffic Administration of the Flemish Community (AWV), using loop detectors. For a period of a couple of weeks, the number of vehicles passing per hour was counted continuously. During the same period, manual counts were done at seven other points during the morning and evening rush-hour. The number of vehicles passing was counted per quarter of an hour, making a distinction between different vehicle categories. The AWV also provided us with reasonable rush-hour traffic data for the E17 highway. Finally the Flemish transport company De Lijn provided us with the timetables of busses, trams and trolleys, which were also checked in situ.

Table 1 gives an overview of the different types of vehicles used in the microsimulation, together with their main simulation properties. The vehicle types were grouped into 4 categories; for each category an OD matrix was constructed for each quarter of an hour, by combination and interpolation of the gathered data. An iter- 
Table 1

Vehicle categories and properties

\begin{tabular}{lllcl}
\hline Vehicle type & Maximum speed $(\mathrm{km} / \mathrm{h})$ & Demand matrix & Proportion & Emission class \\
\hline Car & $50(90)$ & 1 & 10.0 & CAR \\
& $70(110)$ & 1 & 50.0 & CAR \\
Van & $90(130)$ & 1 & 31.6 & CAR \\
Lorry & 80 & 1 & 5.2 & DHV \\
Lorry with trailer & 80 & 1 & 1.3 & MHV \\
Bus & 80 & 1 & 0.1 & MHV \\
Motorcycle & 90 & 1 & 0.8 & MHV \\
City bus & 65 & 1 & 1.0 & MHV \\
Trolleybus & 65 & 2 & 100.0 & MHV \\
Tram & 50 & 3 & 100.0 & MHV \\
\hline
\end{tabular}

The speed values between brackets are used for the vehicles on the E17 highway.

ative process was used to match the link flow intensities to counts. For the first category (demand matrix 1), consisting of several vehicle types, the proportions of each type are given in Table 1. A number of vehicle properties, such as mean vehicle top speed, maximum acceleration and deceleration, and a number of driver properties, such as mean reaction time, awareness and aggressivity, were also introduced in the simulation, to represent the Flemish vehicle fleet and drivers. One has to point out that most microsimulation models tend to maximize the vehicle driving speeds, taking into account the maximum link driving speed and the vehicle top speed. When traffic is freely flowing, this could result in all cars passing by at about the same speed. Therefore we divided the car vehicle type into 3 categories with different maximum speeds, to better represent different driving behaviour; the different percentages in Table 1 for the car type are tuned to obtain the best agreement with the actual situation.

\subsection{Acoustic parameters}

The noise immission simulation requires that some additional acoustic parameters about the vehicles and the environment are defined. Because the Nord 2000 database only distinguishes between three emission classes, the vehicle categories used for traffic modelling were mapped to these three categories, as shown in Table 1. Because no emission values for motorcycles and trams are available up to now, these were considered to have a noise emission comparable with the MHV class, which seemed the best compromise. The information on buildings shown in Fig. 3, to be used in the propagation model, was loaded from GIS. The external walls were assumed to have a normalized impedance of 20 , which results in a power absorption coefficient of about 0.1 for perpendicular incidence. Because of the $2.5 \mathrm{D}$ model, the buildings have a flat roof in the simulation.

For the propagation calculations, a temperature of $21^{\circ} \mathrm{C}$, a relative humidity of $70 \%$ and a wind direction with most probability from the south-west were used, which are typical Flemish values for the time of year when measurements were 
performed. For simulating the ground effects, the ground was considered to be acoustically hard over the full area.

\subsection{Comparison with immission measurements}

On the 5th of June 2003, during the evening rush-hour, sound measurements were done at the 6 observer points shown in Fig. 3. Using a B\&K 2260 Investigator with a B\&K 4189 free-field microphone mounted at a height of $1.2 \mathrm{~m}$ and a distance of 1.2 $\mathrm{m}$ from the edge of the road, time series of $L_{\mathrm{Aeq}, 1 \mathrm{~s}}$ values were measured, for a period of $15 \mathrm{~min}$. Simultaneously, the number of vehicles passing by was counted. The results of these counts are shown in Table 2, together with the number of vehicles obtained from 5 simulation runs, each with different random generator seed values. For the random process involved in the simulation, these numbers illustrate the variation between different runs, which is mostly small. Overall for the evening rushhour, the simulated number of vehicles differs by at most about $25 \%$ from the actual counts, which is small enough to have no significant effect on equivalent noise levels. Measured $L_{\text {Aeq, } 15 \mathrm{~m}}$ values and statistical noise levels are shown in Table 3.

Traffic simulation and emission calculation at the evening rush-hour of our coded network resulted in a 15 min time-series of vehicle emissions; a single run with a timestep of $100 \mathrm{~ms}$ was used. For reasons of efficiency, the propagation calculation was done for each observer point seperately, and was in itself also divided into three parts:

1. For the vehicles within a range of $150 \mathrm{~m}$ of the observer in question, exact positions of the sources were used. This covered about 10-20 vehicles each timestep for which directional emission values could be used. The beamtrace model was set to account for a maximum of 4 reflections and 1 edge diffraction.

2. To make propagation calculations feasible and efficient for the traffic at larger distance, a grid of emission points was used with a spacing of $5 \mathrm{~m}$. The same beamtrace configuration was used.

Table 2

Vehicle counts at the observer points and results of different simulation runs

\begin{tabular}{lcccccccc}
\hline Point & Measurement & Run 1 & Run 2 & Run 3 & Run 4 & Run 5 & Mean & Deviation \\
\hline 1 (a) & 46 & 39 & 36 & 46 & 38 & 38 & 39.4 & $-14.3 \%$ \\
1 (b) & 64 & 72 & 62 & 77 & 67 & 77 & 71.0 & $+10.9 \%$ \\
2 (a) & 27 & 36 & 33 & 35 & 30 & 33 & 33.4 & $+23.7 \%$ \\
2 (b) & 9 & 7 & 8 & 10 & 8 & 9 & 8.4 & $-6.7 \%$ \\
3 (a) & 67 & 79 & 80 & 78 & 72 & 84 & 78.6 & $+17.3 \%$ \\
3 (b) & 62 & 57 & 61 & 59 & 61 & 64 & 60.4 & $-2.6 \%$ \\
4 (a) & 31 & 23 & 33 & 31 & 29 & 28 & 28.8 & $-7.1 \%$ \\
4 (b) & 27 & 26 & 28 & 25 & 27 & 23 & 25.8 & $-4.4 \%$ \\
5 (a) & 246 & 208 & 160 & 205 & 216 & 197 & 197.2 & $-19.8 \%$ \\
6 (a) & 286 & 280 & 255 & 281 & 298 & 266 & 276.0 & $-3.5 \%$ \\
\hline
\end{tabular}

For points 1-4, vehicles were counted along the measurement side of the road (a), and in the opposite travelling direction (b). The counts are each for a duration of $15 \mathrm{~min}$, and include all vehicle types under consideration. The mean value is also given, together with its deviation from the measurement. 
Table 3

Measurements and simulations - statistical levels and power spectrum features for a period of $15 \mathrm{~min}$

\begin{tabular}{|c|c|c|c|c|c|c|c|c|c|c|c|c|}
\hline \multirow[t]{2}{*}{ Point } & \multicolumn{6}{|c|}{ Measurement } & \multicolumn{6}{|c|}{ Simulation } \\
\hline & $L_{\text {Aeq }}$ & $L_{5}$ & $L_{50}$ & $L_{95}$ & $\alpha$ & $\epsilon$ & $L_{\text {Aeq }}$ & $L_{5}$ & $L_{50}$ & $L_{95}$ & $\alpha$ & $\epsilon$ \\
\hline 1 & 70.4 & 76.7 & 63.5 & 49.3 & -0.23 & 0.36 & 67.5 & 74.3 & 60.9 & 50.3 & 0.01 & 0.39 \\
\hline 2 & 64.4 & 70.1 & 53.5 & 43.6 & -0.22 & 0.28 & 65.6 & 73.5 & 51.4 & 34.0 & 0.01 & 0.39 \\
\hline 3 & 67.2 & 73.4 & 58.6 & 49.7 & -0.21 & 0.42 & 66.2 & 73.5 & 58.8 & 49.9 & -0.16 & 0.35 \\
\hline 4 & 66.0 & 72.5 & 56.2 & 41.4 & 0.09 & 0.50 & 66.2 & 74.2 & 51.7 & 34.9 & -0.42 & 0.40 \\
\hline 5 & 73.0 & 78.3 & 69.9 & 62.3 & -0.18 & 0.46 & 71.3 & 75.3 & 68.9 & 56.6 & -0.31 & 0.46 \\
\hline 6 & 73.8 & 77.6 & 71.6 & 60.6 & -0.09 & 0.18 & 73.7 & 77.7 & 70.1 & 62.1 & -0.14 & 0.44 \\
\hline
\end{tabular}

The simulated values are for one simulation run only.

3. Emission of the traffic on the E17 highway was taken into account seperately. A grid with a spacing of $5 \mathrm{~m}$ was also used here, but during beamtrace only propagation in the vertical plane connecting source and receiver (diffraction over the buildings) was included. The noise barriers were not taken into account.

Finally, a time series of $L_{\text {Aeq, } 1 \mathrm{~s}}$ immission values was calculated at each observer point. Fig. 4 shows a 5 min fragment at the first observer point, together with a measurement excerpt. Because of the probabilistic nature of traffic micromodelling, both time-series are not equal, but about the same dynamic pattern seems to arise; the duration and magnitude of the events are also comparable.

Fig. 5 shows the statistical and cumulative level distributions at the 6 observer points, both for measurements and simulations. For the $L_{\mathrm{Aeq}, 15 \mathrm{~m}}, L_{5}$ and $L_{50}$ values, the deviation between simulation and measurement is on the average within $3 \mathrm{~dB}(\mathrm{~A})$, as can be seen in Table 3. The deviation in $L_{95}$ is more striking for points 2, 4 and 5 . The streets in which point 2 and 4 were situated are both streets with low traffic. Between the passing by of vehicles, the simulated level drops to unrealistically low values of $30 \mathrm{~dB}(\mathrm{~A})$ and less for this suburban area. The noise coming from other sources such as the wind, birds, pedestrians and cyclists, planes at high altitude, ventilation
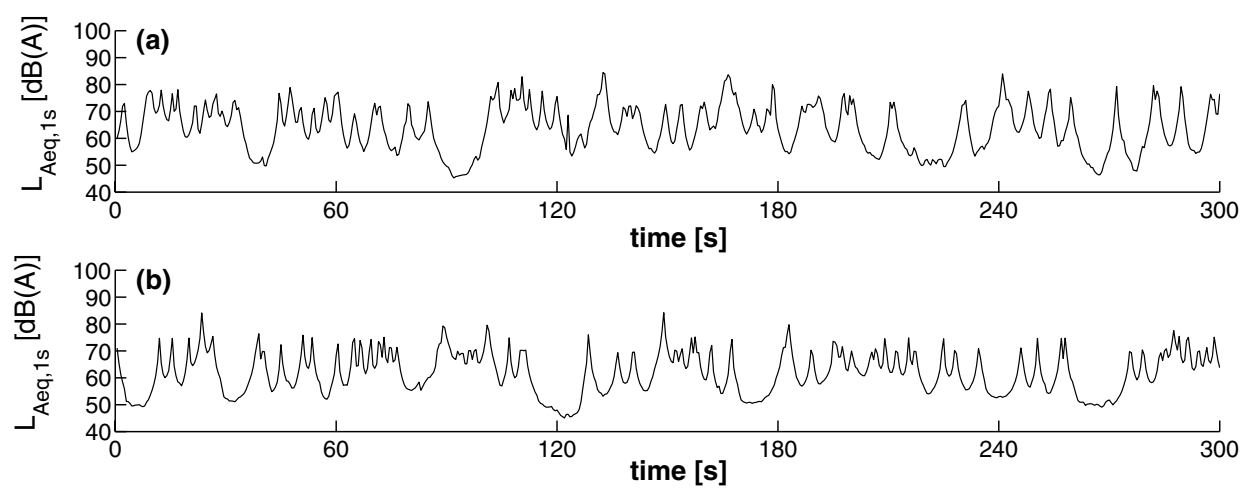

Fig. 4. Time-series of $L_{\text {Aeq, } 1 \mathrm{~s}}$ at the first observer point, for $5 \mathrm{~min}$. (a) Measurement; (b) simulation. 
and cooling systems causes the level not to drop so low in reality. The noise coming from the E17 highway has, because of the distance, no effect on the noise immission levels at these points in our simulation. The road at the fifth observation point carries a high amount of traffic, but because of the presence of a lot of shops in the neighbourhood, also a lot of pedestrians are passing by at the evening rush-hour.

The power spectra of fluctuations in $L_{\text {Aeq, } 1 \mathrm{~s}}$ at the observer points are shown in Fig. 6. To calculate the linear fits in the interval $[0.002 \mathrm{~Hz} ; 0.2 \mathrm{~Hz}]$, the spectra were first logarithmically resampled to 12 values per octave, to assure that the lower
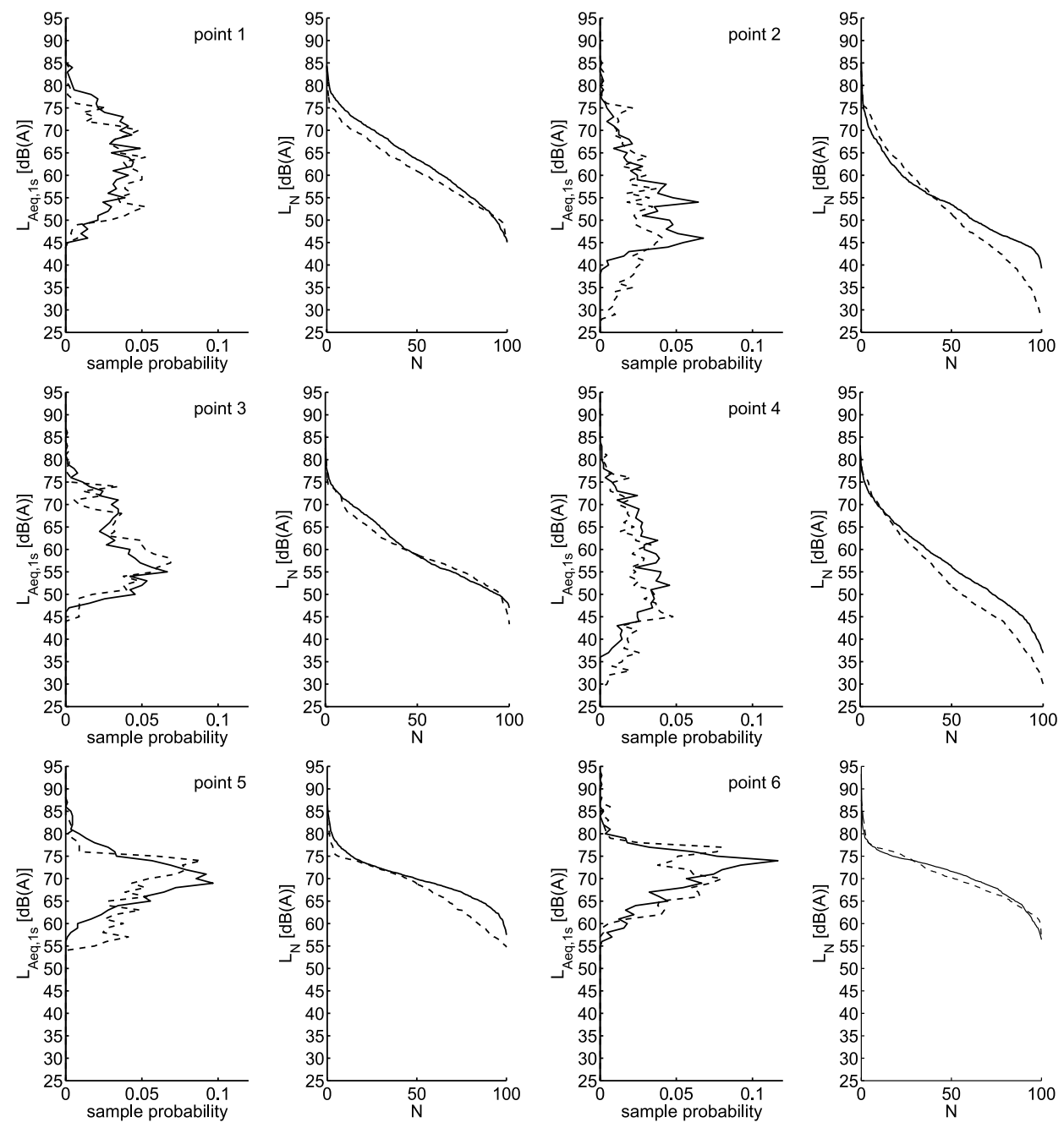

Fig. 5. Statistical and cumulative level distributions at the six observer points. The measurements are in solid lines, the simulations in dashed lines. 
frequencies make an equal contribution on a log-log scale. Values for the slope $\alpha$ and standard deviation from a straight line $\epsilon$ can be found in Table 3. Overall, the spectra are rather flat, indicating an almost random passing of vehicles. One can see that the correspondence for $\alpha$ is best for points 3 and 6 , for which the statistical and cumulative level distribution correspondence was also the best. Comparison between measurements and simulation in this paragraph gives us some confidence in the methodology used.
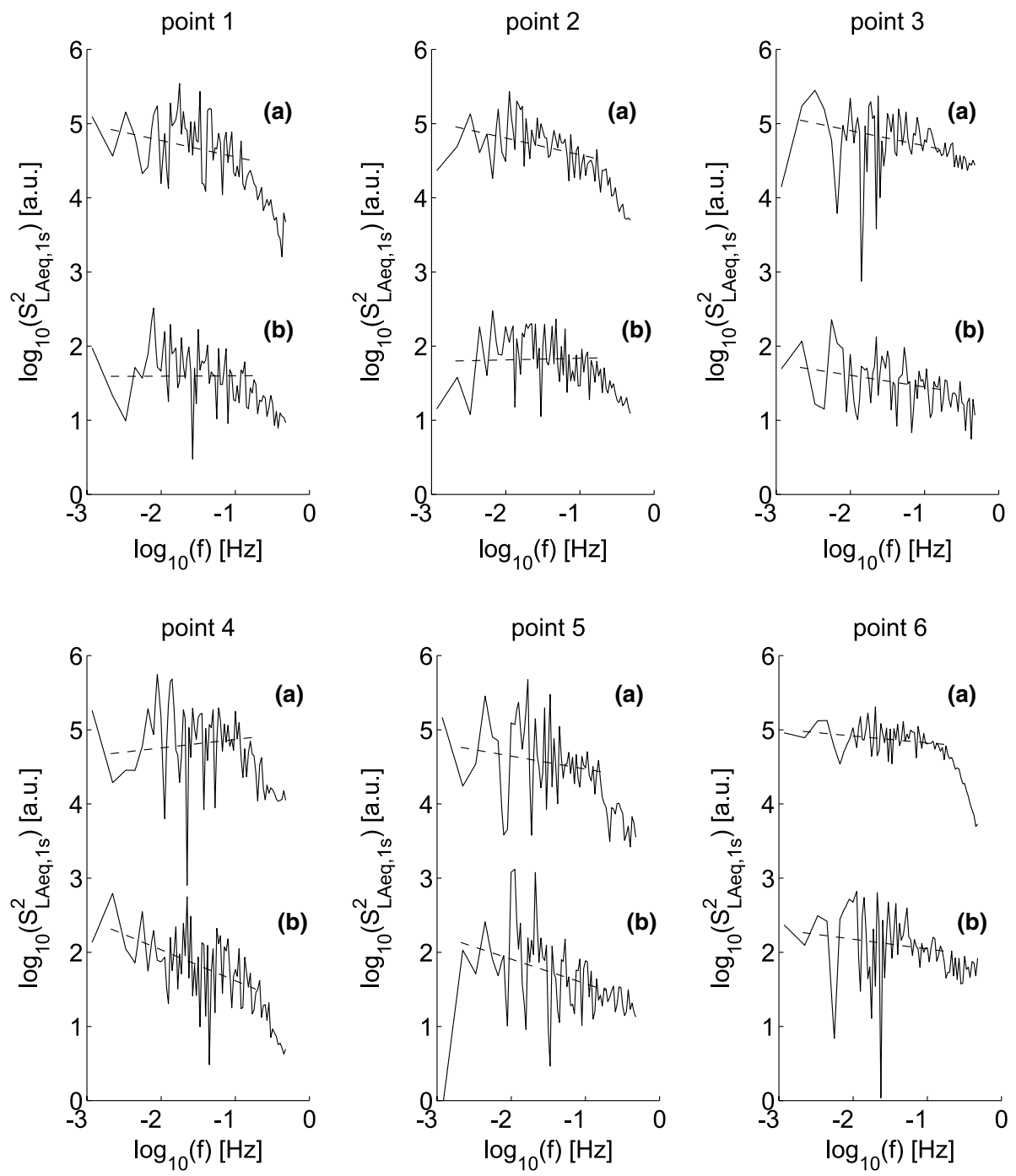

Fig. 6. Power spectrum of fluctuations in $L_{\text {Aeq, } 1 \mathrm{~s}}$ at the six observer points (solid lines), together with a linear fit in the interval $[0.002 \mathrm{~Hz} ; 0.2 \mathrm{~Hz}]$ (dashed lines). (a) Measurement; (b) simulation. 


\subsection{Sound field dynamics maps}

As an example of a map showing the dynamics of the acoustic field, the signalized junction on the main district road between observer points 5 and 6 was chosen. A rectangular grid of $100 \times 100$ observers with a spacing of $1 \mathrm{~m}$ was put around the junction, at a height of $1.2 \mathrm{~m}$. A 15 min time-series of vehicle emissions was calculated using a timestep of $0.5 \mathrm{~s}$. Only noise emission of vehicles within a distance of $300 \mathrm{~m}$ from the center of the grid was taken into account; exact vehicle positions were used. The beamtrace propagation model was set to account for a maximum of 3 reflections and 1 diffraction. The calculation of a dynamic immission map with these settings takes about 60 hours, using an AMD Athlon at 1.7 Ghz.

Fig. 7 shows the resulting maps of $L_{\mathrm{Aeq}, 15 \mathrm{~m}}, L_{5}, L_{5}-L_{95}$ and $\alpha$. The major road, from the north-west to the south-east, carries about 5 times more traffic than the crossing minor road, which is reflected in the signal times: the green (red) time on the major road is $65 \mathrm{~s}(30 \mathrm{~s})$, on the minor road it is $20 \mathrm{~s}(75 \mathrm{~s})$. As a consequence, vehicles are standing still for a much longer period on the minor road. This, together with the discretization of the vehicle positions, characteristic to microsimulation models, explains the dots appearing on the north-east arm of the junction. In this context, one has to point out that the noise emission of vehicles standing still is somehow overestimated using the Nord 2000 database, because only noise measurements of vehicles driving at a non-zero speed are available. In any case, only the observer points along the edge of the road are of interest, for obvious reasons.
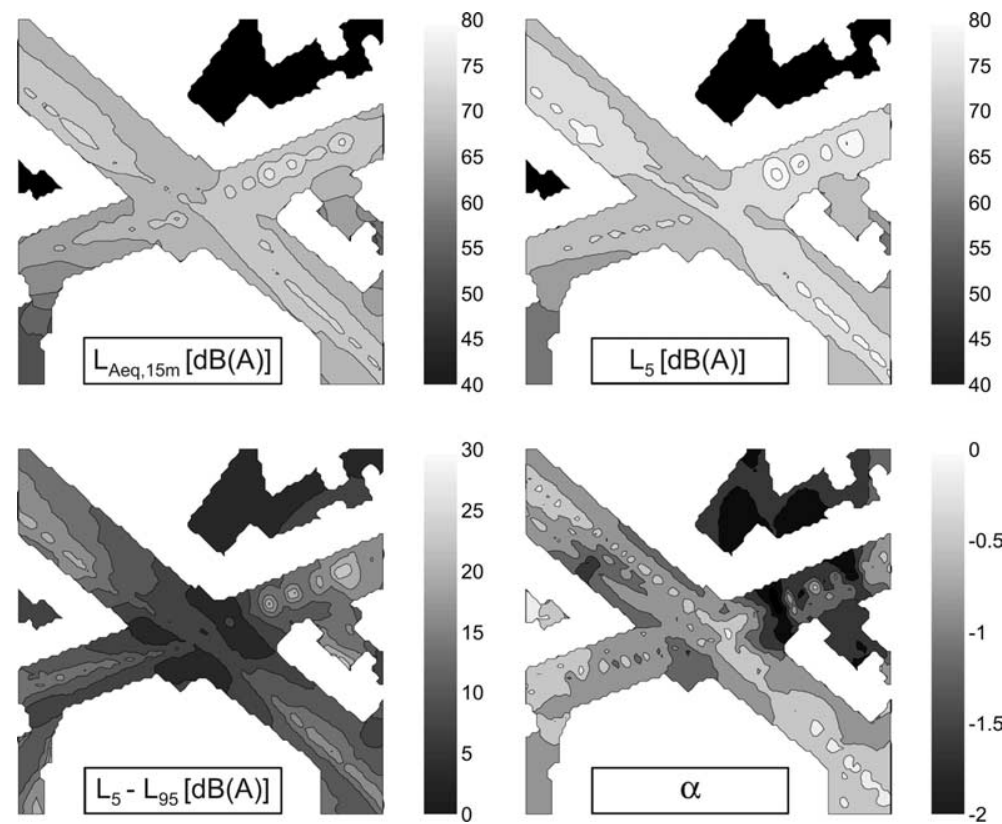

Fig. 7. Noise maps at a signalized junction. The white areas represent the buildings. 
The $L_{\text {Aeq, } 15 \mathrm{~m}}$ and $L_{5}$ maps show little variation along the footpath and façade. The $L_{5}-L_{95}$ map shows much more variation. Low values can be found on the junction itself, because vehicles are passing nearly all the time, so both $L_{5}$ and $L_{95}$ are high. In the backyard shown in the north, $L_{5}-L_{95}$ is also low but this time because $L_{5}$ and $L_{95}$ are both low. In contrast, in the backyards to the east and in the southwest corner high $L_{5}-L_{95}$ is observed. This can be explained by the partial screening: for some vehicle positions, traffic noise can reach these backyards directly. The $\alpha$ map shows a more nuanced image. Values along the major road are almost zero, implying random traffic dynamics. Values along the stoplines, and in particular on the minor road, have a $1 / f^{1.5}$ to $1 / f^{2}$ behaviour, indicating very predictable dynamics caused by stop-and-go traffic. In the backyard in the north, the level fluctuations have a more $1 / f$ to $1 / f^{1.5}$ behaviour, and is now more differentiated from the dynamics on the junction itself.

\section{Influence of traffic flows}

To simulate the effect of a street closure (e.g., because of road repair works further on in the network), the north-eastern link of the junction discussed in the previous section was closed, while leaving the original signal times; the other three arms of the junction still carry traffic. Using the original demand matrices, this resulted in a (minimal) rerouting of the traffic. This way, it was possible to study the dynamics of the noise in the minor arm of the junction, caused by traffic on the major road. Fig. 8(a) shows the resulting $L_{5}-L_{95}$ and $\alpha$ maps. Subsequently, the traffic demand on the major district road was gradually increased. Fig. 8(b) shows the results for a demand $20 \%$ higher than the original demand. The traffic becomes more and more dense, small jams are formed but disappear after some time. Fig. 8(c) shows results for a demand $50 \%$ higher than the original; in this case some small traffic jams do not disappear any more, resulting in a situation where the individual velocities of the vehicles are strongly correlated to each other, and where the capacity of the major road drops.

For the different demands, a small but visible trend can be found on the $L_{5}-L_{95}$ maps in the no-traffic arm of the junction. However, when considering the $\alpha$ maps, a clearer evolution is visible. The original demand, which does not display any jams, results in a rather chaotic behaviour with $\alpha \approx-0.5$. The $20 \%$ higher demand, near the traffic jam transition point, shows a more $1 / f$ behaviour in the closed street; this behaviour is now also found in the northern backyard. When traffic demands are still further increased, the clustering and jamming of the vehicles results in a $1 / f^{1.5}$ to $1 / f^{2}$ behaviour of the noise immission spectrum. At the façades along the major road and away from the junction a $1 / f$ spectrum is still observed. This corresponds with the earlier findings of $1 / f$ behaviour in the power spectrum of traffic flow in microsimulating models near the jamming transition point $[43,44]$, which is reflected here in the noise immission spectrum.

If anything, these few scenario calculations show that taking into account traffic noise dynamics makes noise maps much more sensitive to minor changes in traffic. 
(a)
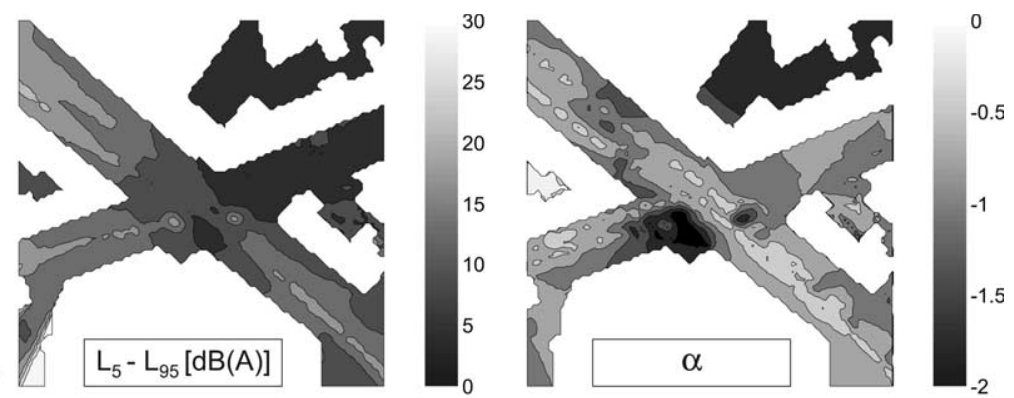

(b)
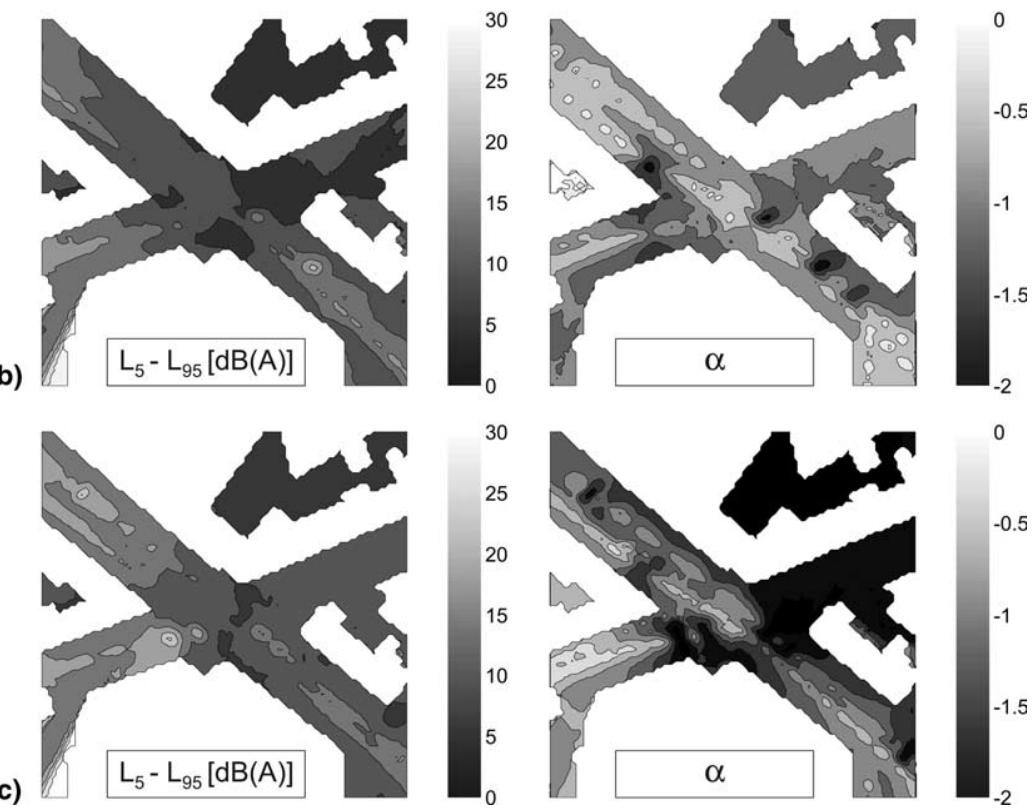

Fig. 8. Maps of $L_{5}-L_{95}$ and $\alpha$ for different scenarios of traffic demand on the main district road, from the north-west to the south-east. (a) Original demand; (b) 20\% higher demand; (c) 50\% higher demand.

\section{Conclusions}

A tool for dynamic traffic noise prediction, based on microsimulation of the traffic in an urban neighbourhood coupled with a state-of-the-art beamtrace propagation model was introduced. The unique feature of this approach is that it allows to estimate the effect of traffic flow management on noise in a much wider area than previous models. The model was compared with measurements of $L_{\mathrm{Aeq}, 1 \mathrm{~s}}$ over 15 min and in general good agreement was found for all the statistical properties of this fluctuating noise level. Further improvement could include the introduction of corrections on the emission of accelerating and decelerating vehicles, the introduction of correct motorcycle and tram emission spectra, and the implementation of diffuse reflections in the propagation model. 
A method for evaluating the patterns in time variations of a series of dynamic noise immission values, based on the power spectrum of sound amplitude fluctuations, was proposed. A combination of this new indicator with more conventional indicators based on statistical noise levels allows to monitor the effect of traffic on the urban soundscape more accurately. Based on these indicators, it was shown that minor changes in traffic (possibly caused by flow management) have a much larger effect on the soundscape than expected on the bases of average emission and immission mapping.

\section{References}

[1] Greenshields B. A study of traffic capacity. In Proceedings of the highway research board, vol. 14, p. 448-477; 1935.

[2] Miedema HME, Vos H. Exposure-response relationships for transportation noise. J Acoust Soc Am 1998;104(6):3432-45.

[3] Borst H. URBIS: instrument for local environmental survey. In Proceedings of the 29th International Congress on Noise Control Engineering (Internoise 2000), Nice, France; 2000.

[4] Li B, Tao S, Dawson RW, Cao J, Lam K. A GIS based road traffic noise prediction model. Appl Acoust 2002;63(6):679-91.

[5] Roberts MJ, Western AW, Webber MJ. A theory of patterns of passby noise. J Sound Vibr 2003;262(5):1047-56.

[6] Viollon S, Lavandier C. Multidimensional assessment of the acoustic quality of urban environments. In Proceedings of the 29th international congress on noise control engineering (Internoise 2000), Nice, France; 2000.

[7] Krebber W, Genuit K, Sottek R. Sound quality of vehicle exterior noise. In Proceedings of Forum Acusticum, Sevilla, Spain; 2002.

[8] Krebber W, Genuit K, Sottek R. Road traffic noise - a matter of sound quality? In Proceedings of the 31st international congress on noise control engineering (Internoise 2002), Dearborn, USA; 2002.

[9] Sottek R, Krebber W, Genuit K. Road traffic noise simulation in urban streets. In Proceedings of the 31st international congress on noise control engineering (Internoise 2002), Dearborn, USA; 2002.

[10] De Coensel B, Botteldooren D, De Muer T. Classification of soundscapes based on their dynamics. In Proceedings of the 8th international congress on noise as a public health problem (ICBEN), Rotterdam, The Netherlands; 2003.

[11] Lavandier C, Barbot B. Influence of the temporal scale on the relevance of acoustic parameters selected to characterize urban sound environments. In Proceedings of the 5th European conference on noise control (Euronoise 2003), Naples, Italy; 2003.

[12] Kokowski P, Makarewicz R. Interrupted traffic noise. J Acoust Soc Am 1997;101(1):360-71.

[13] Makarewicz R, Fujimoto M, Kokowski P. A model of interrupted road traffic noise. Appl Acoust 1999;57(2):129-37.

[14] Rylander R, Björkman M. Road traffic noise influenced by road bumps. J Sound Vibr 2002;250(1):157-9.

[15] To WM, Chan TM. The noise emitted from vehicles at roundabouts. J Acoust Soc Am 2000;107(5):2760-3.

[16] Bérengier M. Acoustical impact of traffic flowing equipments in urban area. In Proceedings of Forum Acusticum, Sevilla, Spain; 2002.

[17] Steele C. A critical review of some traffic noise prediction models. Appl Acoust 2001;62(3):271-87.

[18] Leclercq L, Lelong J. Dynamic evaluation of urban traffic noise. In Proceedings of the 17th international congress of acoustics, Rome, Italy; 2001.

[19] Crocker MJ. Rating measures, descriptors, criteria and procedures for determining human response to noise. Encyclopedia of acoustics, vol. 2. New York: Wiley; 1997. p. 943-65. [chapter 80]. 
[20] Griffiths ID, Langdon FJ. Subjective response to road traffic noise. J Sound Vibr 1968;8(1):16-33.

[21] Robinson DW. The concept of noise pollution level. Technical Report NPL Aero Report AC 38, National Physical Laboratory, Aerodynamics Division, March 1969.

[22] Maffiolo V, Castellengo M, Dubois D. Qualitative judgments of urban soundscapes. In Proceedings of the 28th international congress on noise control engineering (Internoise 1999), Fort Lauderdale, FL, USA; 1999.

[23] Voss R, Clarke J. " $1 / f$ noise" in music and speech. Nature 1975;258:317-8.

[24] Voss R, Clarke J. "1/f noise" in music: music from $1 / f$ noise. J Acoust Soc Am 1978;63(1):258-63.

[25] De Coensel B, Botteldooren D, De Muer T. $1 / f$ noise in rural and urban soundscapes. Acta Acust Acust 2003;89(2):287-95.

[26] Helbing D. Traffic and related self-driven many-particle systems. Rev Mod Phys 2001;73:1067-141.

[27] Lighthill MJ, Whitham JB. On kinematic waves. II: A theory of traffic flow on long crowded roads. Proc R Soc Ser A 1955;229:317-45.

[28] Chandler RE, Herman R, Montroll EW. Traffic dynamics: studies in car following. Operat Res 1958;6(2):165-84.

[29] Prigogine I, Andrews FC. A Boltzmann like approach for traffic flow. Operat Res 1960;8(6):789-97.

[30] Cremer M, Ludwig J. A fast simulation model for traffic flow on the basis of boolean operations. Math Comput Simul 1986;28(4):297-303.

[31] Nagel K, Schreckenberg M. A cellular automaton model for freeway traffic. J Phys I Fr 1992;2(12):2221-9.

[32] http://www.paramics-online.com. Paramics is being developed by Quadstone Ltd.

[33] http://www.delta.dk/nord2000.

[34] Jonasson HG, Storeheier S. Nord 2000. New Nordic prediction method for road traffic noise. Technical Report 2001:10, SP Swedish National Testing and Research Institute; 2001.

[35] Heckbert PS, Hanrahan P. Beam tracing polygonal objects. In Proceedings of the 11th international conference on computer graphics and interactive techniques (SIGGRAPH '84), Minneapolis, USA; 1984.

[36] Funkhouser T, Carlbom I, Elko G, Pingali G, Sondhi M, West J. A beam tracing approach to acoustic modeling for interactive virtual environments. In Proceedings of the 25th international conference on computer graphics and interactive techniques (SIGGRAPH '98), Orlando, USA; 1998.

[37] de Berg M, van Kreveld M, Overmars M, Schwarzkopf O. Computational geometry. Berlin: Springer; 1997.

[38] Guibas L, Stolfi J. Primitives for the manipulation of general subdivisions and the computation of Voronoi diagrams. ACM Trans Graph 1985;4(2):74-123.

[39] ISO 9613-2. Acoustics - attenuation of sound during propagation outdoors - part 2: A general method of calculation. Genève, Switzerland; 1996.

[40] Plovsing B, Kragh J. Nord. Comprehensive outdoor sound propagation model. Propagation in atmosphere without significant refraction. Technical Report AV 1849/00, Danish Electronics, Light and Acoustics (DELTA); 2000.

[41] Department of Transport, Welsh Office. Calculation of road traffic noise. The Stationery Office Books, London; 1988. ISBN 0115508473.

[42] Botteldooren D, Decloedt S, Bruyneel J, Pottie S. Characterisation of quiet areas: subjective evaluation and sound level indices. In Proceedings of the joint 137th meeting of the acoustical society of America and the 2nd convention of the European acoustics association (Forum Acusticum), Berlin, Germany; 1999.

[43] Choi MY, Lee HY. Traffic flow and 1/f fluctuations. Phys Rev E 1995;52(6):5979-84.

[44] Zhang X, Hu G. 1/f noise in a two-lane highway traffic model. Phys Rev E 1995;52(5):4664-8. 